\title{
Evaluation of Statistical Shape Modeling in Quantifying Femoral Morphologic Differences Between Symptomatic and Nonsymptomatic Hips in Patients with Unilateral Femoroacetabular Impingement Syndrome
}

\author{
Timothy C. Keating, M.D., Natalie Leong, M.D., Edward C. Beck, M.D., M.P.H., \\ Benedict U. Nwachukwu, M.D., M.B.A, Alejandro A. Espinoza Orías, Ph.D., \\ Xioaping Qian, Ph.D., Kang Li, Ph.D., and Shane J. Nho, M.D., M.Sc.
}

\begin{abstract}
Purpose: To determine whether statistical shape modeling can detect subtle morphologic differences in the shape of the proximal femur that correlate with clinical findings of unilateral femoroacetabular impingement syndrome. Methods: Patients who had diagnoses of unilateral femoroacetabular impingement syndrome and who had existing computed tomography scans of their pelvises were included. Three-dimensional shape models in the form of triangle meshes were generated from the computed tomography images. Statistical shapes of cam-type and normal hips were compared to identify structural differences. Results: The study included 33 hips in 17 subjects. Of the subjects, $7(41.1 \%)$ were male, and $10(58.9 \%)$ were female. The subjects ranged in age from 17-60 years of age (mean $36.3 \pm 11.0$ years old). The statistical shape modeling found mean shapes and modes after optimizing the groupwise correspondence. Symptomatic hips demonstrated $1 \mathrm{~mm}$ of thickening as compared to the femoral necks of asymptomatic hips, corresponding to cam lesions. Conclusions: Symptomatic cam deformities were an average of $1 \mathrm{~mm}$ more prominent in the femoral neck region as compared to the asymptomatic hips when using statistical shape modeling. The present study provides a proof of the concept that statistical shape modeling can be used to examine and help define cam morphology and that subtle morphologic differences may account for developing femoroacetabular impingement syndrome. Clinical Relevance: Using the methods presented in this study, it would be possible to define cam and pincer morphologies by creating statistical shape models, and this work could potentially lead to the development of a new classification system for femoroacetabular impingement syndrome lesions.
\end{abstract}

\section{Introduction}

$\mathbf{F}$ emoroacetabular impingement syndrome (FAIS) was recently defined as a movement-related clinical hip disorder that represents the symptomatic contact between the proximal femur and acetabulum

From the Department of Orthopedic Surgery, Rush University Medical Center (T.C.K., B.U.N. A.A.E.O., X.Q.), Chicago, Illinois; Department of Orthopedic Surgery, University of Maryland (N.L., S.J.N.), Baltimore, Maryland; Department of Orthopedic Surgery, Wake Forest Baptist Health (E.C.B.), Winston-Salem, North Carolina; and Siemens Digital Industries Software (K.L.), Plano, Texas, U.S.A.

The authors report the following potential conflicts of interest or sources of funding: B.U.N. has received hospitality payments from Stryker, Wright Medical Technology, and Zimmer Biomet, and an educational grant from Smith and Nephew, outside the submitted work. S.J.N. receives research support from AlloSource, Arthrex, Athletico, and DJ Orthopaedics, is on the editoriallgoverning board for the American Journal of Orthopedics, and is a board/committee member for the American Orthopaedic Society of Sports during hip motion. Specifically, FAIS occurs when deformities on the proximal femur and acetabulum dynamically collide with each other, leading to chondrolabral injury and the beginning of a degenerative process that is thought to be a precursor of developing

Medicine and the Arthroscopy Association of North America. Full ICMJE author disclosure forms are available for this article online, as supplementary material.

Received January 15, 2018; accepted November 18, 2019

Address correspondence to Edward C. Beck, M.D., M.P.H., Department of Orthopedic Surgery, Wake Forest Baptist Health, 1 Medical Center Boulevard, Winston-Salem, NC,U.S.A.E-mail: edward.beck@rushortho.com

(C) 2019 THE AUTHORS. Published by Elsevier Inc. on behalf of the Arthroscopy Association of North America. This is an open access article under the CC BY-NC-ND license (http://creativecommons.org/licenses/by-nc-nd/4.0/). 2666-061X/1863

https://doi.org/10.1016/j.asmr.2019.11.005 
early hip osteoarthritis. ${ }^{1-3}$ Diagnostic imaging parameters, such as the alpha angle in the anteroposterior and the Dunn lateral views on plain $x$-rays, can visualize pathologic shape changes at the head-neck junction in many, but not all, hips with FAIS. ${ }^{4-6}$ Characterizing the 3-dimensional (3D) pathomorphology of FAIS is difficult because it is often hard to delineate varying morphologic differences on standard 2-D radiography. Pathologic cam-type lesions in atypical positions along the head-neck junction may be missed initially, leading to the involvement of multiple providers and diagnostic treatments of common extra-articular sources of pain. ${ }^{7}$

Statistical shape modeling (SSM) is a powerful tool that has been used for various medical applications to study 3D anatomy and anatomic variations. ${ }^{7-14}$ In standard SSM, a shape model algorithm is used to match corresponding anatomic landmarks such as the greater trochanter between subjects to analyze the inherent differences in the remaining shapes. From these data, the distribution of 3D shapes can be calculated, and the variations in each surface landmark within the population can be demonstrated. ${ }^{10}$ In orthopedics, SSM has been used to better understand pathoanatomy and create de novo 3D renderings for reconstructing the mandible, spine, wrist, forearm, knee, and shoulder..$^{8-10,12,13,15-17}$ With respect to the proximal femur, SSM has been used to characterize changes seen with slipped capital femoral epiphysis and Legg-Calve-Perthes disease as well as to characterize hip fracture risk in the elderly. ${ }^{9,13}$ The utility of SSM has also been applied to cam lesions and to normal and pathologic hips. ${ }^{7}$ However, this modeling has not been used to compare morphologic differences between the symptomatic and the normal hip in patients with unilateral FAIS.

The use of plain radiographs to evaluate a painful hip for FAIS is the current standard of care, even though 3D pathomorphology of hips with FAIS may be oversimplified or may go unrecognized with 2D imaging modalities. SSM may be used to analyze the morphology of the proximal femur using shape information from 3D axial imaging modalities such as MRI and CT. As such, the purpose of the present study was to determine whether SSM can detect subtle morphologic differences in the shape of the proximal femur that correlate with clinical findings of unilateral FAIS. We hypothesized that hips with FAIS would have a shape significantly different from that of hips without clinically apparent impingement.

\section{Methods}

\section{Study Population}

After receiving approval from the local institutional review board, CT scans of the hips of patients undergoing hip arthroscopy for treatment of FAIS by a single, fellowship-trained orthopaedic surgeon were obtained from our institutional database. Inclusion criteria were patients who had clinical diagnoses of unilateral FAIS as previously described ${ }^{18}$ and who had already had CT scans of their pelvises so as to limit additional radiation exposure. Patients with bilateral FAIS and those with only unilateral hip CTs were excluded from the study. Our diagnostic criteria for a cam lesion was an alpha angle of greater than $55^{\circ}$ on either anteroposterior or Dunn lateral views of the hip, as previously stated in the literature. ${ }^{18}$ All patient data were deidentified. The same CT scan protocol had been used for all patients (iliac crest to just below the knee, $3.75 \mathrm{~mm}$ axial slice thickness). Patients' scans selected did not include any signs of current or previous fractures, arthritis, bone cysts, or tumors.

\section{Study Design}

The present retrospective, CT-based image-processing study was designed to investigate the anatomic variance of the proximal femur. First, 3D shape models were generated in the form of triange meshes from the CT images. Then, 2 steps were conducted for the analysis: 1) training set data preparation; and 2) correspondence optimization and statistical analysis.

\section{D Shape Model Generation and Analysis}

The CT scans of the proximal femur were segmented and converted to stereolithography models using commercial segmentation software (version 16, Materialise Mimics, Leuven, Belgium). A gradient-based optimization approach was used; the statistical shape modeling was cast as a problem of seeking optimal correspondence across the set of femurs. All calculations were performed in Matlab R2013a (The MathWorks, Natick, MA, U.S.A), and custom-written Matlab routines were used to produce the SSM. To prepare the training set required for the correspondence optimization algorithm used in this work, the triangle meshes underwent raw data denoising, mesh smoothing and decimation, feature identification (Fig 1), orientation and mesh cutting, iterative closest point alignment (Fig 2) and, finally, B-spline fitting. Points on the femoral head fovea and lesser trochanter were merged by using a validated 3D-3D registration method (accuracy $0.1 \mathrm{~mm}$ in translation, $0.2^{\circ}$ in rotation). ${ }^{19}$

\section{Results}

Of 39 patients with bilateral hip CTs, 17 patients had unilateral FAIS and were included in the final analysis. Of 34 total hips, 17 were symptomatic, and 16 were asymptomatic. One of the control hips was symptomatic due to a mixed or unclear diagnosis and was excluded from the analysis. Of the 17 patients included, $7(41.1 \%)$ were male. They ranged in age from 17-60 and were, on average, $36.3 \pm 11.0$ years old $(95 \%$ CI 


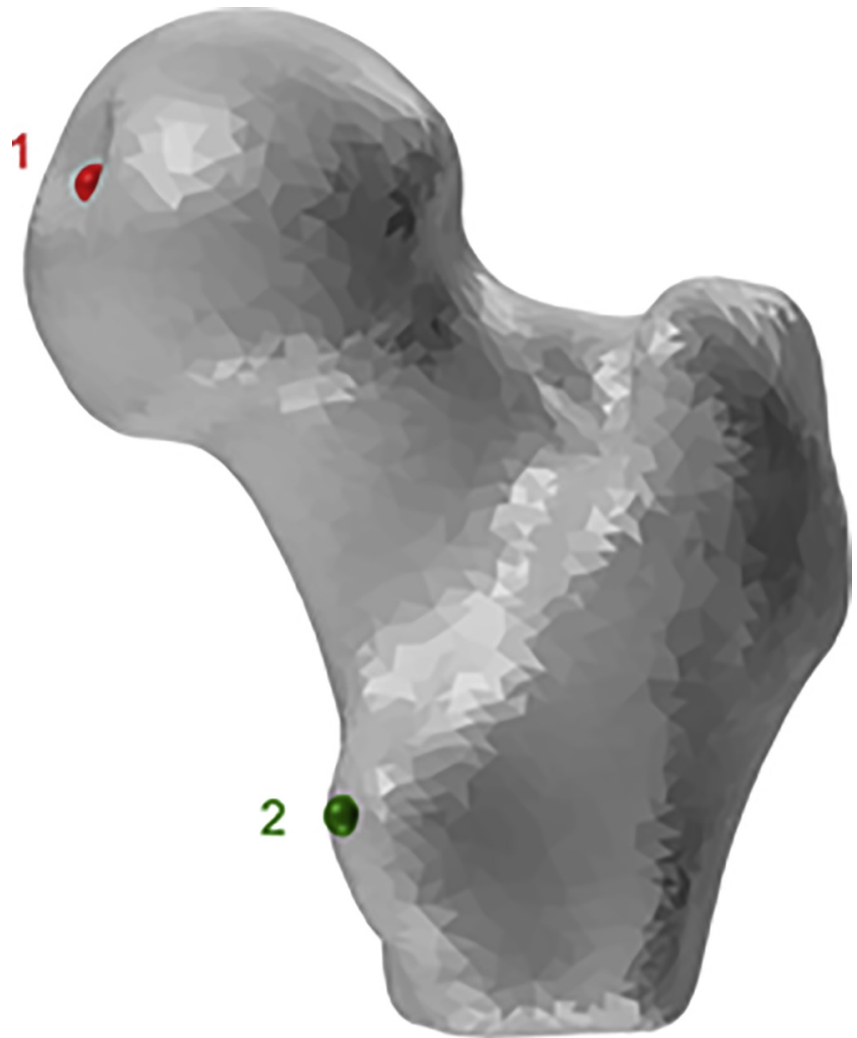

Fig 1. Landmark identification. Statistical shape modeling relies on matching common points to overlay multiple 3D meshes for the identification and quantification of 3D differences. Our study selected the femoral head fovea (1) and lesser trochanter (2) as anatomic landmarks for further processing, as shown in this right-sided femur.

31.0-41.5). The statistical shape modeling found mean shapes and modes after optimizing the groupwise correspondence. The color fields in Fig 3 represent the differences in shape from the mean shape, with darker colors corresponding to greater prominence. Comparing the symptomatic group shown in Fig $3 \mathrm{~B}$ to the asymptomatic group in Fig 3A, a thickening of the femoral neck measuring $1 \mathrm{~mm}$ larger than the mean asymptomatic femoral neck can be seen.

\section{Discussion}

The main finding of the study was that the statistical shape analysis was able to detect a thickening pattern on the femur neck, consistent with the cam deformity, and to show that this deformity is more pronounced in hips that go on to develop FAIS. This work has demonstrated the applicability of SSMs to aid in diagnostic imaging and characterization of a cam deformity. In addition to demonstrating characteristics of FAIS in symptomatic hips, this work also contributes a methodology for establishing baseline normal models of non-FAIS femoral geometry.
In this study, we found that symptomatic cam deformities were an average of $1 \mathrm{~mm}$ more prominent in the femoral neck region as compared to the asymptomatic hip. Overall, the difference between hips with cam impingement and asymptomatic hips was not as large as the 2.5-3.0 mm cited by Harris et al. ${ }^{7}$ This could be accounted for by differences in the patient population seen at each individual center, as well as by differences in sample size and the fact that the present study did not include any hips of patients who were seen by a surgeon for a diagnosis other than FAIS. Additionally, there are differences in the SSM techniques used; the present study employed a correspondence-optimization technique based on smooth geometry, whereas the Harris study used an entropy-minimizing optimization technique based on discrete geometry, which may be prone to larger variances.

The present study used the opposite asymptomatic hip as the control hip; however, it is known that approximately $15 \%-20 \%$ of patients with FAIS have bilateral involvement, ${ }^{20}$ and it is possible that there are morphologic features typical of cam impingement on the asymptomatic side in some patients. Even so, we were able to detect a $1 \mathrm{~mm}$ difference in the thickness of the femoral neck between the asymptomatic and symptomatic hips; this difference might be magnified if patients with subtle FAIS on the asymptomatic side were somehow excluded from the analysis. Because cam pathology is often bilateral, we suspect that if we compared the symptomatic hips in this study to matched hips in control patients without FAIS in either hip, the difference in femoral neck thickness would be greater, and that guiding surgical resection would be more useful with this technique.

\section{Limitations}

There are a number of limitations that need to need to be addressed. First, although combined acetabular overcoverage and cam lesion is the most common type of bony pathology associated with FAIS, the current study did not examine the acetabulum. It is possible that variation in acetabular version could influence differences in femoral morphology, even among hips with cam impingement. Second, a number of variables were not accounted for that could have affected the results, including age, gender and bony morphology. As previous studies have pointed out, there are differences in alpha angles when comparing males and females. ${ }^{21}$ Third, our study size was limited due to the number of patients with bilateral hip CTs that were required for modeling between symptomatic and nonsymptomatic hips. Fourth, as mentioned previously, this study did not compare femoral morphology with patients without FAIS due to the inherent limitation of accessing bilateral hip CTs of healthy controls. 


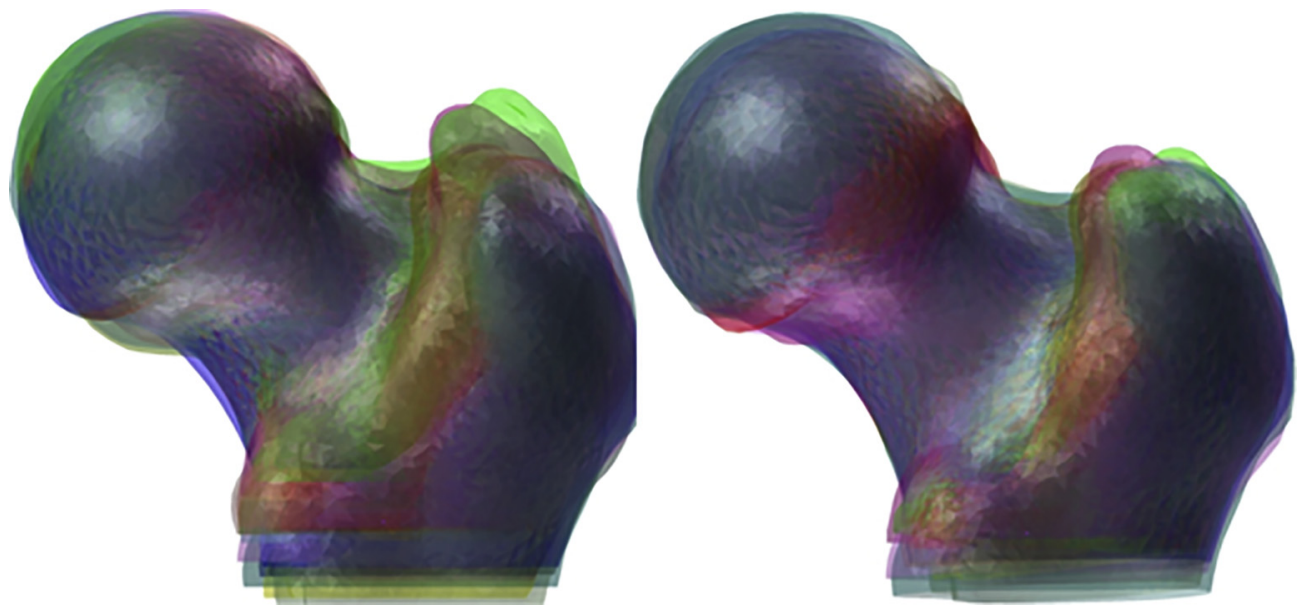

Fig 2. Iterative closest point alignment. Statistical shape modeling is able to overlay accurately 3D meshes from different samples in order to detect even subtle shape differences. Mesh alignment before (left) and after (right) application of the iterative closest point algorithm are shown in this right-sided femur.

It is possible that non-FAIS patients have, on average, different femoral morphology and more significant differences as compared to patients with FAIS. Furthermore, it is also possible that some of the patients in the final analysis had bilateral cams with unilateral symptoms at the time of the study and may have progressed to presenting with contralateral symptoms at a later time.
Fig 3. Three-dimensional heat map. In the 4 images corresponding to a right-sided femur, differences in shape are displayed, with orange being no different and separate colors representing more or less prominence. The average shape of asymptomatic femurs (A) shows that the head-neck junction is relatively consistent among samples. Comparing the shape of symptomatic to asymptomatic femurs (B) shows that the anterosuperior portion of the head-neck junction tends to be more prominent in symptomatic hips, consistent with the most common location of a cam lesion.

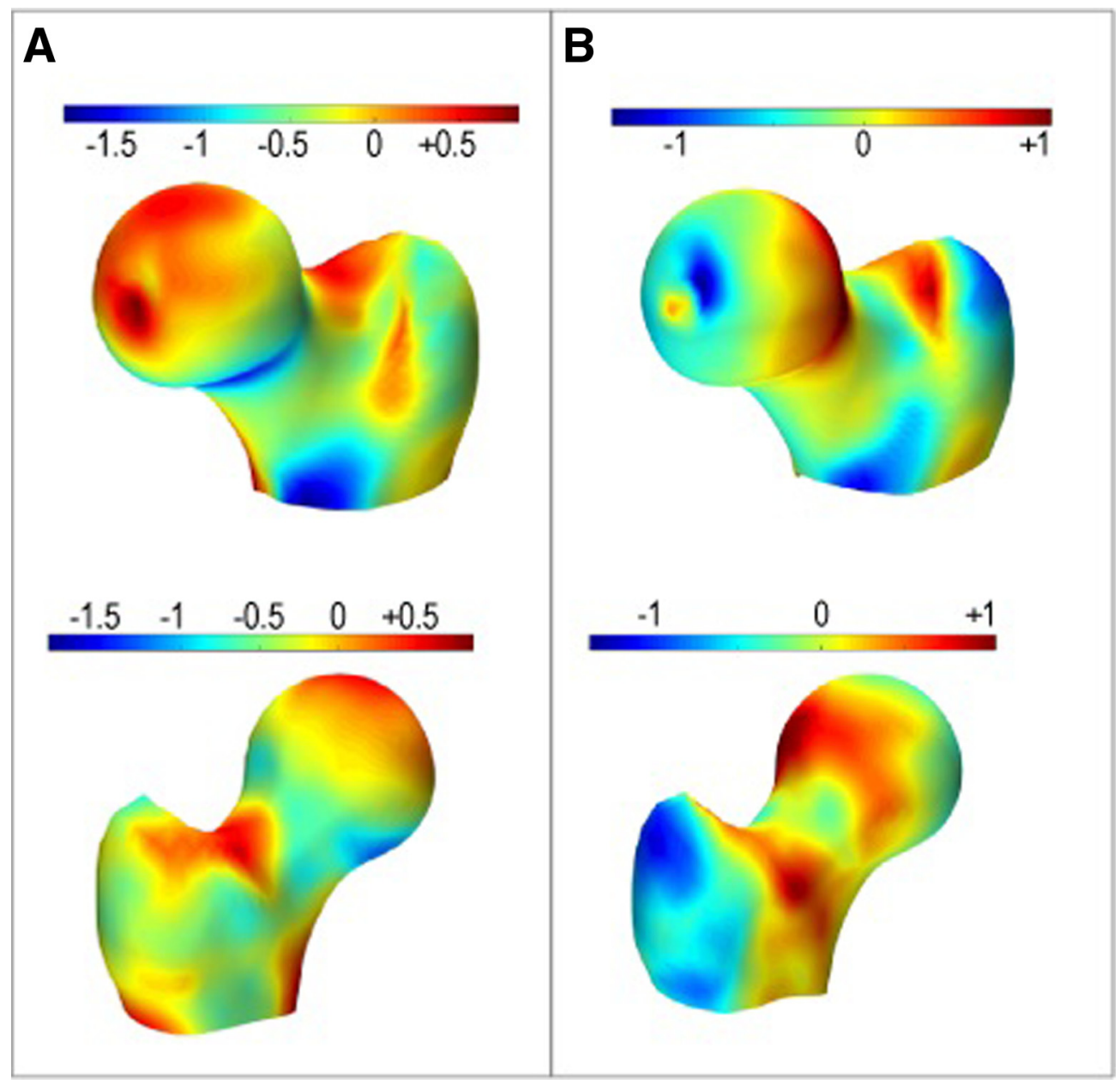




\section{Conclusions}

Symptomatic cam deformities were an average of 1 $\mathrm{mm}$ more prominent in the femoral neck region than in the asymptomatic hips when using SSM. The present study provides a proof of concept that SSM can be used to examine and help to define cam morphology and that subtle morphologic differences may account for developing FAIS.

\section{References}

1. Leunig M, Ganz R. Femoroacetabular impingement: A common cause of hip complaints leading to arthrosis. Unfallchirurg 2005;108(9-10):12-17.

2. Ganz R, Parvizi J, Beck M, Leunig M, Notzli H, Siebenrock KA. Femoroacetabular impingement: A cause for osteoarthritis of the hip. Clin Orthop Relat Res 2003;417: 112-120.

3. Beck M, Kalhor M, Leunig M, Ganz R. Hip morphology influences the pattern of damage to the acetabular cartilage: Femoroacetabular impingement as a cause of early osteoarthritis of the hip. J Bone Joint Surg Br 2005;87: 1012-1018.

4. Clohisy JC, Carlisle JC, Beaule PE, et al. A systematic approach to the plain radiographic evaluation of the young adult hip. J Bone Joint Surg Am 2008;90:47-66 (Suppl 4).

5. Notzli HP, Wyss TF, Stoecklin CH, Schmid MR, Treiber K, Hodler J. The contour of the femoral head-neck junction as a predictor for the risk of anterior impingement. $J$ Bone Joint Surg Br 2002;84:556-560.

6. Siebenrock KA, Ferner F, Noble PC, Santore RF, Werlen S, Mamisch TC. The cam-type deformity of the proximal femur arises in childhood in response to vigorous sporting activity. Clin Orthop Relat Res 2011;469: 3229-3240.

7. Harris MD, Datar M, Whitaker RT, Jurrus ER, Peters CL, Anderson AE. Statistical shape modeling of cam femoroacetabular impingement. J Orthop Res 2013;31: 1620-1626.

8. Campbell JQ, Petrella AJ. Automated finite element modeling of the lumbar spine: Using a statistical shape model to generate a virtual population of models. J Biomech 2016;49:2593-2599.

9. Chan EF, Farnsworth CL, Klisch SM, Hosalkar HS, Sah RL. 3-dimensional metrics of proximal femoral shape deformities in Legg-Calve-Perthes disease and slipped capital femoral epiphysis. J Orthop Res 2018;36: 1526-1535.
10. Coogan JS, Kim DG, Bredbenner TL, Nicolella DP. Determination of sex differences of human cadaveric mandibular condyles using statistical shape and trait modeling. Bone 2018;106:35-41.

11. Corouge I, Dojat M, Barillot C. Statistical shape modeling of low level visual area borders. Med Image Anal 2004;8: 353-360.

12. Pedoia V, Lansdown DA, Zaid M, et al. Three-dimensional MRI-based statistical shape model and application to a cohort of knees with acute ACL injury. Osteoarthrit Cartil 2015;23:1695-1703.

13. Taghizadeh E, Chandran V, Reyes M, Zysset $P$, Buchler P. Statistical analysis of the inter-individual variations of the bone shape, volume fraction and fabric and their correlations in the proximal femur. Bone 2017;103:252-261.

14. Ward AD, Hamarneh G. Statistical shape modeling using MDL incorporating shape, appearance, and expert knowledge. Med Image Comput Comput Assist Interv 2007;10:278-285.

15. Baumbach SF, Binder J, Synek A, et al. Analysis of the three-dimensional anatomical variance of the distal radius using 3D shape models. BMC Med Imaging 2017;17:23.

16. Mauler F, Langguth C, Schweizer A, et al. Prediction of normal bone anatomy for the planning of corrective osteotomies of malunited forearm bones using a threedimensional statistical shape model. J Orthop Res 2017;35:2630-2636.

17. Poltaretskyi S, Chaoui J, Mayya M, et al. Prediction of the pre-morbid 3D anatomy of the proximal humerus based on statistical shape modelling. Bone Joint J 2017;99-B: 927-933.

18. Griffin DR, Dickenson EJ, O'Donnell J, et al. The Warwick Agreement on femoroacetabular impingement syndrome (FAI syndrome): An international consensus statement. Br J Sports Med 2016;50:1 169-1176.

19. Riff AJ, Weber AE, Keating TC, et al. Mirror image modeling of acetabular rim thickness differences in patients with unilateral femoroacetabular impingement syndrome. Arthros Sport Med Rehab 2019;1:el-e6.

20. Klingenstein GG, Zbeda RM, Bedi A, Magennis E, Kelly BT. Prevalence and preoperative demographic and radiographic predictors of bilateral femoroacetabular impingement. Am J Sports Med 2013;41:762-768.

21. Hooper P, Oak SR, Lynch TS, Ibrahim G, Goodwin R, Rosneck J. Adolescent femoroacetabular impingement: Gender differences in hip morphology. Arthroscopy 2016;32:2495-2502. 